\title{
Discrete ARMA Model Applied for Tumor Growth Inhibition Modeling and LQR-based Chemotherapy Optimization
}

\author{
Sotirios G. LiLIOPOULOS, GEORGE S. STAVRAKAKIS \\ School of Electrical and Computer Engineering Technical University of Crete, \\ Chania, GREECE
}

\begin{abstract}
Mathematical models for tumor growth inhibition (TGI) are an important tool in the battle against cancer allowing preclinical evaluation of potential anti-cancer drugs and treatment schedules. However, most of these models are nonlinear and their structure is based on complex hypotheses. Therefore, tumor growth mathematical models with simple structure and minimal number of parameters could be of great importance. In this article, an autoregressive moving average (ARMA) model for cancer tumor growth and equivalent its state space representation are estimated, presented and evaluated based on laboratory data of TGI in mice. The proposed model was proven capable of describing with accuracy the tumor growth under single-agent chemotherapy. At the same time, an optimal control problem was formulated to identify optimal drug dosages for the tumor eradication. The linear quadratic regulator (LQR) controller was used with success in optimizing both periodic and intermittent chemotherapy treatment schedules reducing the tumor mass while keeping dosages under acceptable toxicity.
\end{abstract}

Keywords - Cancer, tumor growth inhibition (TGI), TGI ARMA model, optimal control, linear quadratic regulator (LQR), optimized periodic chemotherapy, intermittent chemotherapy

Received: April 2, 2021. Revised: August 11, 2021. Accepted: August 21, 2021. Published: August 31, 2021.

\section{Introduction}

Despite the major improvements in medicine, cancer remains one of the deadliest diseases. Chemotherapy is one of the main treatments against it. However, trying to find an equilibrium between the efficacy of the chemo-treatment and its side effects is a particularly challenging task. To this direction, many mathematical models have been proposed in the literature [1]-[3] trying to capture the tumor growth dynamics, its response to the treatment (i.e. chemotherapy, immunotherapy) and ultimately to provide a better understanding of its mechanics. These models can be used as a base for in-silico designs and evaluations of chemotherapy regimens during pre-clinical tests and clinical trials [2]. In addition, the use of these models in combination with optimal control theory [3]-[7] may also provide personalized treatment and lead to optimal chemotherapy dose schedules with minimum side effects.

Even though such models provide good approximations of tumor's growth, many of them are complex and therefore they are difficult to understand and to be used. Their complexity derives not only from their large number of biologically relevant parameters, but also from their effort to model specific processes and phenomena [2], [3] that take place in a malignant tumor's microenvironment. A different approach to model the growth of a solid tumor under treatment is to consider its progression as a time series. Time series models can have many forms and have been extensively used for forecasting. Autoregressive moving average (ARMA) models [8] are one of the most popular tools to analyze data. This kind of systems are relatively simple in implementation and have been used in numerous applications, e.g., electricity load forecasting [9], electroencephalogram (EEG) analysis [10], applications in economy [8], etc.

In this paper, a new approach to describe tumor growth inhibition (TGI) under the effect of single anti-cancer drugs is introduced. An ARMA system which models the growth of solid tumors is identified and evaluated using laboratory data of TGI experiments in mice [11]. Then, linear quadratic regulator (LQR)-based optimal control of tumor dynamics is used to explore possible optimal chemotherapy dosages for both periodic and intermittent treatment schedules. Finally, the simulations results are presented, discussed, and conclusions are drawn.

\section{Materials and Methods \\ 2.1 Data Acquisition}

Tumor weight data was derived from the experimental studies in human-to-mouse cancer xenografts reported by Bilalis et al. [11]. Fragments of AsPC-1 human pancreatic cancer cells were subcutaneous transplanted in male NOD/SCID mice. Gemcitabine was administered intraperitonously (i.p.) at dose levels of $100 \mathrm{mg} / \mathrm{kg}$ on days 19 and 26 after the inoculation time (day 0 ).

\subsection{ARMA-models Backround}

ARMA models have a long history in forecasting and time series analysis. Despite that, they have not been used extensively to model cancer's growth, until now. An ARMA model is a combination of two linear models, the autoregressive (AR) and moving average (MA) models, respectively [8]. It can be defined as a linear input-output model which uses a weighted linear combination of past values of inputs and data observations (i.e. backfit to historical data) to perform predictions. An ARMA model can be described by (1):

$$
w_{k}=\sum_{i=1}^{p} a_{i} w_{k-i}+\sum_{j=1}^{q} b_{j} u_{k-j}, \quad k \in \mathbb{Z},
$$

where $w_{k}$ is the prediction (output of the model), $w_{k-i}, i \leq p$ are the past observations and $u_{k-j}, j \leq q$ are the past inputs, with $p \leq q$. The set of parameters $(p, q)$ defines the order of the ARMA model. Parameters $a_{i} \in \mathbb{R}$ and $b_{j} \in \mathbb{R}$ are weights associated with each previous observation and input, respectively. In the present work, $w$ and $u$ correspond to the tumor weight observations and chemotherapy dosages, respectively. 


\subsection{LQR Optimal Control [12],[13]}

The linear quadratic regulator (LQR) is a commonly used method that provides optimal state-feedback laws for linear systems. This practically enables closed-loop stability and high-performance design of systems. Given a discrete-time linear system of the following form:

$$
\begin{aligned}
x[k+1] & =A x[k]+B u[k] \\
y[k] & =C x[k]
\end{aligned}
$$

where $A \in \mathbb{R}^{n \times n}, B \in \mathbb{R}^{n \times m}, C \in \mathbb{R}^{l \times n}, x[k] \in \mathbb{R}^{n}$ is the system state vector, $u[n] \in \mathbb{R}^{m}$ is the input to this system (i.e. the chemotherapy drug amount) and $y[k] \in \mathbb{R}^{l}$ is the output (i.e. the tumor weight) at time $k, \mathrm{LQR}$ tries to determine a control input that minimizes a performance index. The quadratic cost function is a typical form of this index and it is described by the following equation:

$$
J=\sum_{k=0}^{\infty}\left(x^{T}[k] Q x[k]+u^{T}[k] R u[k]\right)
$$

where $Q$ and $R$ are the real positive semi-definite and positive definite weighting matrices for each state $x$ and the control variable $u$, respectively. The selection of $Q$ and $R$ matrices is not unique. There are many combinations which ensure the stability of the system and the optimal closed-loop performance. Even though it is a common practice to be selected through trial-and-error, sometimes this might be time consuming and therefore more sophisticated techniques such as the one proposed by Pouliezos [12] can be used.

The cost function $J$ is minimized using the state-feedback controller described by the equation below:

$$
u[k]=-R^{-1} B^{T} S x[k] \triangleq-K x[k]
$$

where $K=R^{-1} B^{T} S$ is the optimal feedback gain and $S$ is a positive definite symmetric matrix and the unique solution of the algebraic Riccati equation (ARE), below:

$$
A^{T} S+S A-S B R^{-1} B^{T} S+Q=0 .
$$

The uniqueness of the ARE solution is implied by the controllability and observability of $(A, B)$ and $(A, C)$, respectively [13].

\section{Results and Discussion \\ 3.1 Model Development}

A crucial step when fitting an ARMA model to data (i.e. tumor weight progression) is the identification of its order, $(p, q)$. ARMA models of each possible order $(p, q)$, with $0<$ $p \leq 5$ and $0<q \leq 5$ were fitted each time to the observed tumor growth data of [8]. The method of linear interpolation was used to approximate the tumor weight values in the time instants (i.e. days) that a measurement was not performed. Model parameters were estimated by using the nonlinear optimization Neural Network Algorithm (NNA) proposed by Sadollah et al. [14], while the order of the model was selected based on the Akaike information criterion (AIC) [8]. Based on AIC, an ARMA model of order $(3,3)$ described by the following equation was selected:

$$
\begin{aligned}
w_{k} & =a_{1} w_{k-1}+a_{2} w_{k-2}+a_{3} w_{k-3}+ \\
& +b_{1} u_{k-1}+b_{2} u_{k-2}+b_{3} u_{k-3}
\end{aligned}
$$

where $a_{1}=1.8812, a_{2}=-0.6371, a_{3}=-0.2443$ and $b_{1}=b_{2}=-10^{-5}, b_{3}=-17.847$ are the estimated ARMA coefficients. In Fig. 1 below, the model-fitted tumor weight curve is plotted against the observed tumor growth data of [11]. It can be observed that the model was able to describe accurately the observed tumor growth under gemcitabine treatment. This fact can be also confirmed by the small values of the evaluation metrics, such as the root mean square error (RMSE) and the mean absolute percentage error (MAPE) (see Table I).

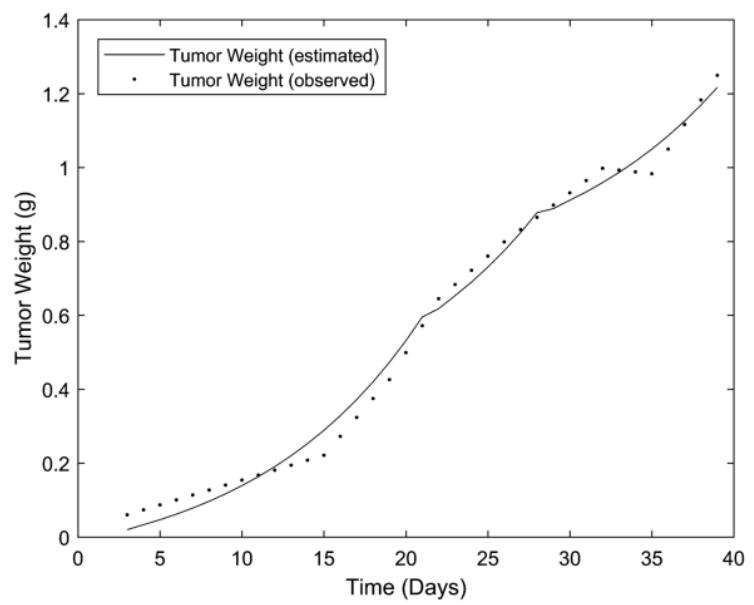

Fig. 1. Observed/interpolated $(\bullet)$ and best fitted $(3,3)$ model tumor growth curve obtained in mice given i.p. doses of gemcitabine $(100 \mathrm{mg} / \mathrm{kg}$ on days 19 and 26).

TABLE I. ARMA $(3,3)$ MODEL'S FITTING ERRORS

\begin{tabular}{|c|c|c|c|}
\hline Model order $(\mathbf{p}, \mathbf{q})$ & AIC & RMSE $\mathbf{g}$ & MAPE (\%) \\
\hline$(3,3)$ & -244.92 & 0.0302 & 12.31 \\
\hline
\end{tabular}

\subsection{LQR Optimal Control Treatment}

Optimal administration of chemotherapy drugs is a challenging problem. A solution to this problem can be accomplished with LQR controllers. Through linear state feedback, LQR can achieve closed-loop optimal control of the anti-cancer drug dose levels while at the same time eliminating the tumor. To do that, the system of (7) is necessary to be converted in a state-space form. This is feasible by calculating its transfer function $H(z)$. Generally, the transfer function of a system can be transformed to a nonunique state-space representation using a discrete-time realization algorithm (DRA) [13]. The transfer function of (7) can be calculated as follows:

$$
\begin{gathered}
\begin{array}{c}
w[k]= \\
a_{1} w[k-1]+a_{2} w[k-2]+a_{3} w[k-3] \\
+b_{1} u[k-1]+b_{2} u[k-2]+b_{3} u[k-3]
\end{array} \\
\begin{array}{c}
W(z)=W(z)\left(a_{1} z^{-1}+a_{2} z^{-2}+a_{3} z^{-3}\right)+U(z)\left(\mathrm{b}_{1} z^{-1}\right. \\
\left.\quad+b_{2} z^{-2}+b_{3} z^{-3}\right)
\end{array} \\
H(z)=\frac{W(z)}{U(z)}=\frac{b_{1} z^{-1}+b_{2} z^{-2}+b_{3} z^{-3}}{1-a_{1} z^{-1}-a_{2} z^{-2}-a_{3} z^{-3}} \\
H(z)=\frac{-10^{-5} z^{-1}-10^{-5} z^{-2}-17.847 z^{-3}}{1-1.8812 z^{-1}+0.6371 z^{-2}+0.2443 z^{-3}}
\end{gathered}
$$

Among several equivalent state-space forms of the above transfer function, the observable canonical form ensures the 
observability of the derived system [13]. The state-space representation of $(8)$ in observable canonical form is described by the equations below:

$$
\begin{aligned}
x[k+1] & =\left[\begin{array}{ccc}
0 & 1 & 0 \\
0 & 0 & 1 \\
-a_{3} & -a_{2} & -a_{1}
\end{array}\right] x[k]+\left[\begin{array}{l}
b_{1} \\
b_{2} \\
b_{3}
\end{array}\right] u[k] \\
y[k] & =\left[\begin{array}{lll}
1 & 0 & 0
\end{array}\right][k]
\end{aligned}
$$

Although the derived state-space system is not stable, it is both observable and controllable. Thus, it is feasible to perform TGI LQR optimal control based on it. The weighting matrices $Q$ and $R$ were selected as $\operatorname{diag}\{0.010 .01\}$ and $10^{4}$, respectively [12].

While the main goal of the optimal control problem is to eradicate the tumor, it is particularly important this to be done with the minimum cost, i.e. minimal side effects. High dose levels may lead to acute toxicity on healthy cells and severe side effects. To avoid such phenomena, it is necessary to impose hard constraints on the system's control variable $u$, i.e. the chemotherapy dosage. Therefore, when the proposed by the LQR controller dose level exceeds a predefined threshold, the control signal is constrained by the following inequality:

$$
u_{\min } \leq u \leq u_{\max }
$$

where $u_{\min }=0$ and $u_{\max }$ are the minimum and the maximum dose levels, respectively. In order to balance toxicity and chemo treatment efficacy, $u_{\max }$ was set to 200 $\mathrm{mg} / \mathrm{kg}$ (i.e. $5.4 \mathrm{e}-3 \mathrm{~g}$ for mouse body weight of $27 \mathrm{~g}$ ). The initial values of the states are $x[0]=\left[x_{1}(0), x_{2}(0), x_{3}(0)\right]^{T}=$ $[0.02,0.0334,0.0468]$ (obtained from the laboratory measurements) and the optimal feedback gain matrix is $K=$ $[0.0039,0.0135,-0.0189]$, derived by applying the MATLAB's dlqr function.

Several cases of different periodic treatment schedules were examined. More specifically, optimal doses of gemcitabine for five different treatment schedules were explored. In the first case, i.e. Case 1, the controller calculated doses for continuous (i.e. every day) drug administration. For the rest of the cases, i.e. cases 2, 3, 4 and 5 the controller calculated optimal drug doses for periodic treatments (see Table II).

TABLE II. PERIODIC LQR TREATMENT RESULTS

\begin{tabular}{|c|c|c|c|c|}
\hline Cases & Dose schedule & $\boldsymbol{u}_{\text {total }}(\mathrm{mg} / \mathrm{kg})$ & $\boldsymbol{y}_{\max }(\mathrm{g})$ & $\boldsymbol{y}_{\text {zero }}$ (days) \\
\hline Case 1 & Continuous & 50.57 & 0.0659 & 23 \\
\hline Case 2 & every 2 days & 58.46 & 0.0924 & 29 \\
\hline Case 3 & every 3 days & 68.24 & 0.1256 & 35 \\
\hline Case 4 & every 5 days & 97.67 & 0.2226 & 46 \\
\hline Case 5 & every 7 days & 148.21 & 0.3900 & 57 \\
\hline
\end{tabular}

Gemcitabine was administered every 2, 3, 5 and 7 days for the Cases 2, 3, 4 and 5, respectively, until the tumor's eradication achievement. The response of the state-space system (i.e. the tumor growth in g) along with the optimal control input (i.e. the gemcitabine dose levels in $\mathrm{mg} / \mathrm{kg}$ ) for each case are shown in Fig. 2-6. In all cases, the chemotherapy treatment begins at day 3 with dose administrations in the range of 10 to $15 \mathrm{mg} / \mathrm{kg}$. Then, the estimated dose levels are gradually decreased until the tumor is fully eradicated at the $57^{\text {th }}$ day in Case 5. Moreover, it can be clearly seen that increasing the interval between the doses, leads to increased dose levels proposed by the controller. Particularly, in the cases 4 and 5 (see Fig. 5 and 6) the control signal (i.e. dose levels) initially increases linearly until it reaches a plateau and then decreases until the tumor is fully eradicated. The total amount of the drug $u_{\text {total }}$ required to inhibit the tumor growth is also increased. The same happens for the treatment duration $y_{\text {zero }}$. It is also important to note that in all these cases studied the proposed dosages did not exceed the defined hard bounds.
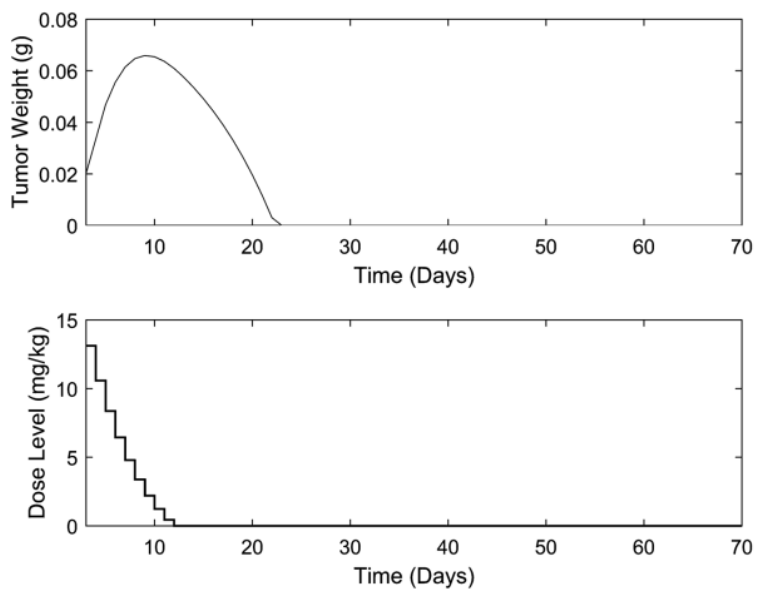

Fig. 2. System's response (i.e. tumor weight) and optimal control input (i.e. dose level). Continuous treatment (Case 1).
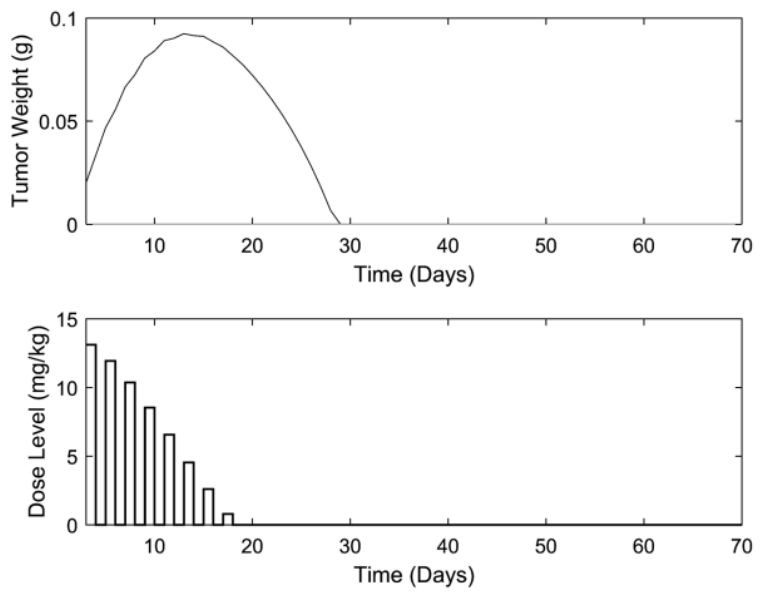

Fig. 3. System's response (i.e. tumor weight) and optimal control input (i.e. dose level). Periodic treatment of (1-2) days (Case 2).
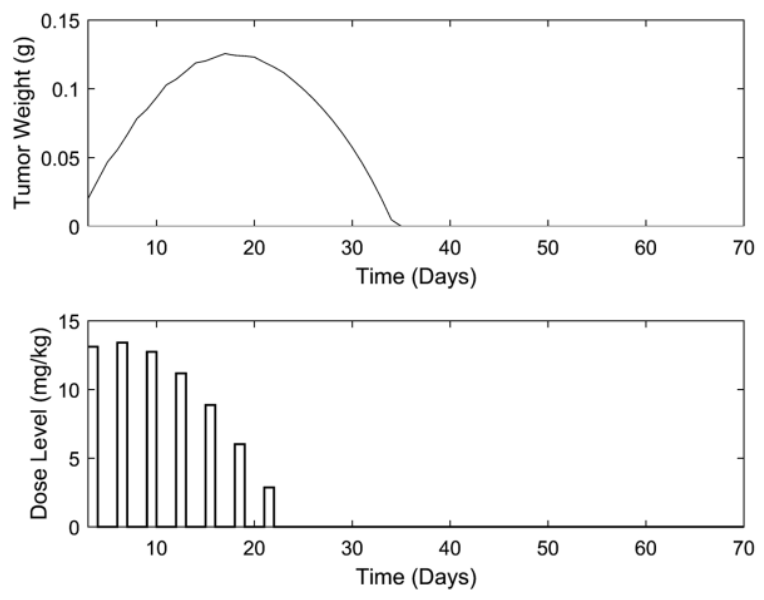

Fig. 4. System's response (i.e. tumor weight) and optimal control input (i.e. dose level). Periodic treatment of (1-3) days (Case 3). 

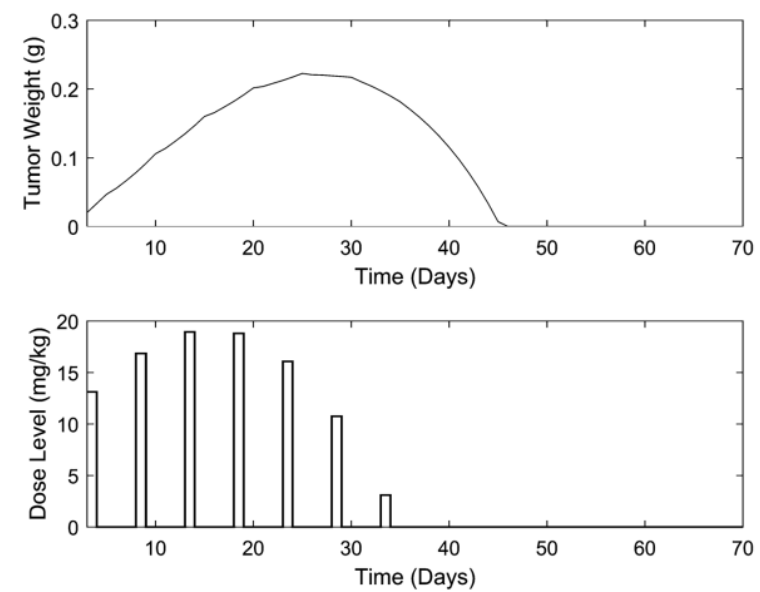

Fig. 5. System's response (i.e. tumor weight) and optimal control input (i.e. dose level). Periodic treatment of (1-5) days (Case 4).
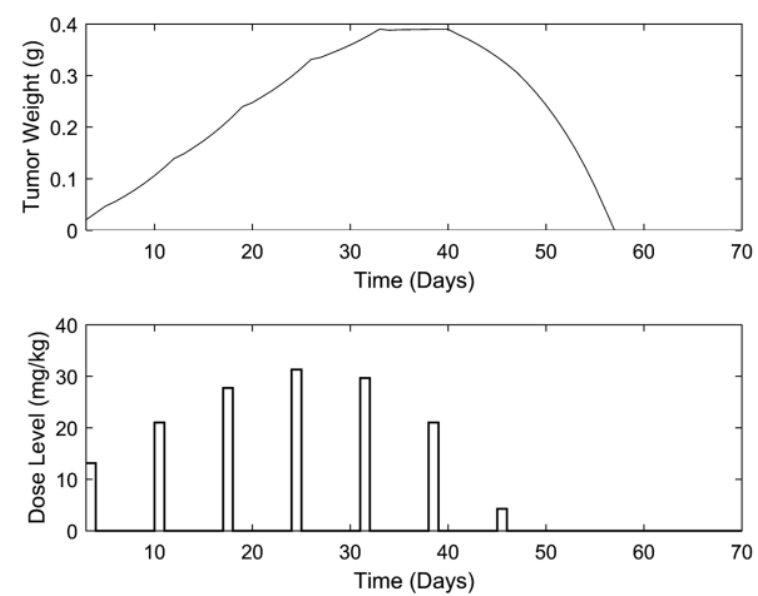

Fig. 6. System's response (i.e. tumor weight) and optimal control input (i.e. dose level). Periodic treatment of (1-7) days (Case 5).

Whereas in the abovementioned cases the tumor was successfully eliminated, chemotherapy resistant scenarios might occur due to the long exposure to the drug. Cancer chemotherapy resistance is a phenomenon where the neoplastic cells develop the ability to evade the effects of the chemotherapeutic treatment, leading to failure in drug response [15], [16]. To this direction, optimal drug dosages for intermittent chemotherapy schedules were also explored. To be more precise, optimal dosages for two cases of different intermittent treatment schedules were investigated. In both cases chemotherapy was administered every 3 days for four times, i.e. $\mathrm{q} 3 \mathrm{dx} 4$. Then, to avoid toxicity and drug resistance, the treatment paused for a period of $t r_{p}=7$ and 14 days, respectively. Finally, it is restarted with dose administrations every 5 days for four times, i.e. q5dx4. A summary of the results is presented in Table III. The tumor weight curves and the optimal dosages are shown in Fig. 7-8. It can be observed that in all cases the tumor size was minimized in a period of $\sim 50$ days.

TABLE III. INTERMITTENT LQR TREATMENT RESULTS

\begin{tabular}{|c|c|c|c|c|c|}
\hline Cases & Dose schedule & $\begin{array}{c}\boldsymbol{t r}_{\boldsymbol{p}} \\
\text { (days) }\end{array}$ & $\begin{array}{c}\boldsymbol{u}_{\text {total }} \\
(\mathrm{mg} / \mathrm{kg})\end{array}$ & $\begin{array}{c}\boldsymbol{y}_{\max } \\
(\mathrm{g})\end{array}$ & $\begin{array}{c}\boldsymbol{y}_{\text {zero }} \\
\text { (days) }\end{array}$ \\
\hline Case 6 & $\mathrm{q} 3 \mathrm{dx} 4, \mathrm{q} 5 \mathrm{dx} 4$ & 7 & 71.27 & 0.1394 & 43 \\
\hline Case 7 & $\mathrm{q} 3 \mathrm{dx} 4, \mathrm{q} 5 \mathrm{dx} 4$ & 14 & 85.63 & 0.1908 & 52 \\
\hline
\end{tabular}
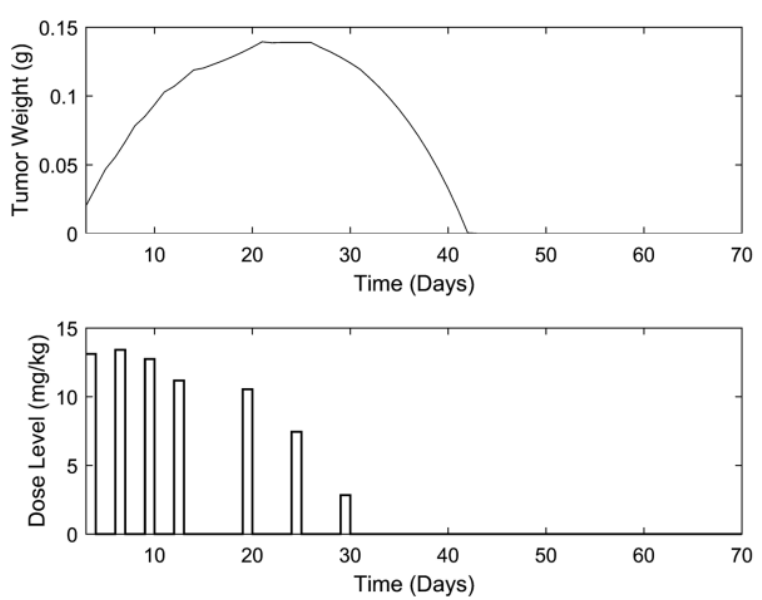

Fig. 7. System's response (i.e. tumor weight) and optimal control input (i.e. dose level). Intermittent treatment (Case 6).
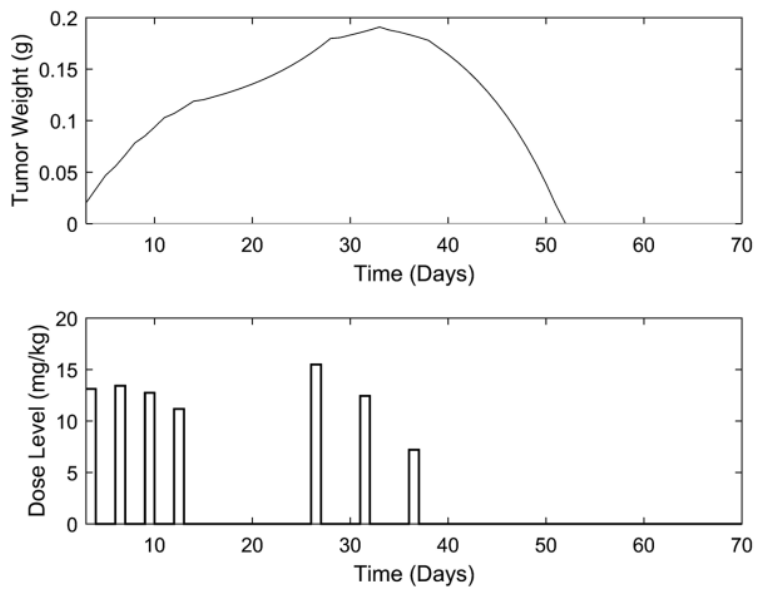

Fig. 8. System's response (i.e. tumor weight) and optimal control input (i.e. dose level). Intermittent treatment (Case 7).

Another important observation is that when increasing the length of chemotherapy holidays, the total amount of drug is also increasing. Despite that, these treatment pauses may act positively for the patient by significantly reducing the adverse effects of toxicity and consequently improving their quality of life [17], [18].

\section{Conclusion}

Anti-cancer chemotherapy is a heavy systemic treatment. This means that chemo drugs travel through the patient's bloodstream and they might affect not only cancerous cells at the tumor site but also the healthy ones. Therefore, prolonged exposure, in combination with high levels of drug concentrations might cause acute toxicity and side effects. Mathematical modeling of tumor growth along with optimal control theory application may provide a solution towards this problem.

In this work, a new approach to model tumor growth under chemotherapy treatment was presented. More specifically, an ARMA $(3,3)$ model for cancer growth under chemotherapy along with its equivalent state space representation were developed, estimated using laboratory data and evaluated. A model of this form does not require knowledge of the drug's pharmacokinetics (PK); thus, it is easier to be implemented and used. The results showed an excellent fit to the tumor weight data of [11], under the effect of gemcitabine in mice. 
Then, the optimal control theory through the LQR control method was used to determine optimal dose regimens that can minimize the tumor size. Furthermore, to avoid extreme levels of toxicity hard bounds to the drug dosages were applied. In all such cases studied, i.e. continuous and periodic drug administration, the tumor was annihilated in an acceptable time period. However, in cases where the treatment duration is long and the dose levels are high, the patient might face significant side effects. Intermittent treatment schedules are an alternative solution which can offer reduced toxicity and improve the patient's quality of life. To this direction, several optimal intermittent chemotherapy schedules were explored. In all these cases, the cancerous cells were successfully eradicated while at the same time the exposure to the drug was minimized and the introduced chemotherapy holidays help the patient to avoid severe side effects.

In conclusion, the use of estimated (based on laboratory data) linear mathematical models of TGI and LQR optimal control in the fight against cancer is proven essential. The presented methodology can provide oncologists with computational tools to design optimal and patient-specific chemotherapy schedules to confront cancer successfully, while improving the quality of life of the patients. Studies have shown that metronomic chemotherapy $(\mathrm{mCHT})$ (i.e. the continuous or frequent administration of chemo drugs at low dosages) may be a promising strategy to control tumor growth [19]-[21]. For this reason, scientists should focus on the identifying optimal metronomic schedules using not only optimal control but also artificial intelligence and machine learning algorithms [21]. Last but not least, further research has to be done to explore optimal treatment schedules not only for cancer monotherapies but also for multi-drug regimens which have been proven to be more effective.

\section{Acknowledgment}

The authors would like to thank I. Brellas for his thoughtful comments that helped to improve the manuscript.

\section{References}

[1] A. M. Jarrett et. al, "Mathematical models of tumor cell proliferation: A review of the literature," Expert Review of Anticancer Therapy, vol. 18, pp. 1271-1286, 2018.

[2] A. Yin, D. J. A. R. Moes, J. G. C. Hasselt, J. J. Swen and H. Guchelaar, "A review of mathematical models for tumor dynamics and treatment resistance evolution of solid tumors," CPT: Pharmacometrics \& Systems Pharmacology, vol. 8, pp. 720-737, 2019.

[3] L. G. De Pillis and A. Radunskaya, "The dynamics of an optimally controlled tumor model: A case study," Mathematical and Computer Modelling, vol. 37, pp. 1221-1244, 62003.

[4] N. Alam, M. Sultana, M. S. Alam, M. A. Al-Mamun and M. A. Hossain, "Optimal intermittent dose schedules for chemotherapy using genetic algorithm," ADCAIJ: Advances in Distributed Computing and Artificial Intelligence Journal, vol. 2, pp. 37-52, 2013.
[5] N. Babaei and M. U. Salamci, "Mixed therapy in cancer treatment for personalized drug administration using model reference adaptive control," European Journal of Control, vol. 50, pp. 117-137, 2019.

[6] M. Itik, M. U. Salamci and S. P. Banks, "Optimal control of drug therapy in cancer treatment," Nonlinear Analysis, Theory, Methods and Applications, 2009.

[7] I. S. Mavromatakis, S. G. Liliopoulos and G. S. Stavrakakis, "Optimized intermittent pharmaceutical treatment of cancer using nonlinear optimal control techniques," WSEAS Transactions on Biology and Biomedicine, vol. 17, pp. 67-75, 2020, doi: 10.37394/23208.2020.17.9.

[8] G. E. P. Box, G. M. Jenkins, G. C. Reinsel and G. M. Ljung, Time Series Analysis: Forecasting and Control, 5th ed. Hoboken, New Jersey: John Wiley \& Sons, Inc, 2016.

[9] Y. Chakhchoukh, P. Panciatici and L. Mili, "Electric load forecasting based on statistical robust methods," in IEEE Transactions on Power Systems, vol. 26, no. 3, pp. 982-991, Aug. 2011, doi: 10.1109/TPWRS.2010.2080325.

[10] V. Sakkalis et al., "Parametric and nonparametric EEG analysis for the evaluation of EEG activity in young children with controlled epilepsy," Computational Intelligence and Neuroscience, vol. 2008, pp. 1-15, 2008.

[11] P. Bilalis et. al, "Self-healing $\mathrm{pH}-$ and enzyme stimuli-responsive hydrogels for targeted delivery of gemcitabine to treat pancreatic cancer," Biomacromolecules, vol. 19, pp. 3840-3852, 92018.

[12] A. Pouliezos, Montern Control Theory, Athens, Greece: Hellenic Academic Libraries Link, 2015.

[13] K. Ogata, Modern Control Engineering, 5th ed. Upper Saddle River, New Jersey: Prentice Hall, 2010.

[14] A. Sadollah, H. Sayyaadi and A. Yadav, "A dynamic metaheuristic optimization model inspired by biological nervous systems: Neural network algorithm," Applied Soft Computing, vol. 71, pp. 747-782, 10 2018.

[15] S. Raguz and E. Yagüe, "Resistance to chemotherapy: new treatments and novel insights into an old problem," Br J Cancer, vol. 99, pp. 387391, 2008, doi: 10.1038/sj.bjc.6604510.

[16] K. O. Alfarouk et. al, "Resistance to cancer chemotherapy: failure in drug response from ADME to P-gp," Cancer Cell International, vol. 15, p. 71, 122015.

[17] T. Onishi, T. Sasaki and A. Hoshina, "Intermittent chemotherapy is a treatment choice for advanced urothelial cancer," Oncology, vol. 83, pp. 50-56, 2012

[18] S. R. Berry, R. Cosby, T. Asmis, K. Chan, N. Hammad, and M. K. Krzyzanowska, "Continuous versus intermittent chemotherapy strategies in metastatic colorectal cancer: a systematic review and meta-analysis," Annals of oncology, vol. 26, no. 3, pp. 477-485, 2015, doi: 10.1093/annonc/mdu272.

[19] V. Wichmann, N. Eigeliene, J. Saarenheimo, and A. Jekunen, "Recent clinical evidence on metronomic dosing in controlled clinical trials: a systematic literature review," Acta Oncol., vol. 59, no. 7, pp. 775-785, 07 2020, doi: 10.1080/0284186X.2020.1744719.

[20] M. E. Cazzaniga et al., "Treating advanced breast cancer with metronomic chemotherapy: what is known, what is new and what is the future?," Onco. Targets. Ther., vol. 12, pp. 2989-2997, 04 2019, doi: 10.2147/OTT.S189163.

[21] M. E. Cazzaniga, N. Cordani, S. Capici, V. Cogliati, F. Riva, and M. G. Cerrito, "Metronomic Chemotherapy," Cancers, vol. 13, no. 9, pp. 2236, 05 2021, doi: 10.3390/cancers13092236.

\section{Creative Commons Attribution License 4.0 (Attribution 4.0 International, CC BY 4.0)}

This article is published under the terms of the Creative Commons Attribution License 4.0 https://creativecommons.org/licenses/by/4.0/deed.en US 